\title{
MD1003 (High-Dose Pharmaceutical-Grade Biotin) for the Treatment of Chronic Visual Loss Related to Optic Neuritis in Multiple Sclerosis: A Randomized, Double-Blind, Placebo-Controlled Study
}

\author{
Ayman Tourbah $^{1,2}$ - Olivier Gout ${ }^{3} \cdot$ Alain Vighetto $^{4,5} \cdot$ Véronique Deburghgraeve $^{6}$. $^{2}$ \\ Jean Pelletier ${ }^{7,8}$ - Caroline Papeix $^{9}$ - Christine Lebrun-Frenay ${ }^{10}$ • Pierre Labauge $^{11}$. \\ David Brassat $^{12}$ - Ahmed Toosy ${ }^{13}$ - David-Axel Laplaud ${ }^{14,15}$ - Olivier Outteryck ${ }^{16}$. \\ Thibault Moreau $^{17}$ - Marc Debouverie $^{18}$ - Pierre Clavelou $^{19}$ • Olivier Heinzlef ${ }^{20}$. \\ Jérôme De Sèze ${ }^{21} \cdot$ Gilles Defer $^{22} \cdot$ Frédéric Sedel $^{23}$ • Carl Arndt ${ }^{24}$
}

Published online: 28 May 2018

(C) The Author(s) 2018

\begin{abstract}
Background Chronic visual loss is a disabling feature in patients with multiple sclerosis (MS). It was recently shown that MD1003 (high-dose pharmaceutical-grade biotin or hdPB) may improve disability in patients with progressive MS.
\end{abstract}

Electronic supplementary material The online version of this article (https://doi.org/10.1007/s40263-018-0528-2) contains supplementary material, which is available to authorized users.

Ayman Tourbah

atourbah@chu-reims.fr

1 Department of Neurology, Faculty of Medicine, CHU de Reims, URCA, Reims, France

2 LPN EA 2027, Université Paris 8, Saint-Denis, France

3 Department of Neurology, Fondation Ophtalmologique Adolphe de Rothschild, Paris, France

4 Lyon Neuroscience Research Center (CRNL), ImpAct, INSERM U1028, CNRS, UMR5292, Lyon 1 University, Lyon, France

5 Department of Neurology, Hopital Neurologigue, Hospices Civils de Lyon, Bron, France

6 Department of Neurology, CHU de Rennes, Rennes, France

7 Department of Neurology, APHM, Hôpital de la Timone, Marseille, France

8 UMR 7339, CRMBM, CNRS, Aix-Marseille Université, Marseille, France

9 Department of Neurology, GH Pitié Salpêtrière, Paris, France

10 Department of Neurology Pasteur 2, Université Nice Cote d'Azur, Nice, France
Objective The aim of this study was to evaluate whether MD1003 improves vision compared with placebo in MS patients with chronic visual loss.

Methods The MS-ON was a 6-month, randomized, doubleblind, placebo-controlled study with a 6-month open-label extension phase. Adult patients with MS-related chronic visual loss of at least one eye [visual acuity (VA) below 0.5 decimal chart] were randomized 2:1 to oral MD1003 $300 \mathrm{mg} /$ day or placebo. The selected eye had to show worsening of VA within the past 3 years following either

11 Department of Neurology, CHU de Montpellier, Montpellier, France

12 INSERM U1043, Centre de Resource et de Competence SEP, Hopital Pierre Paul Riquet, Université de Toulouse,

Toulouse, France

13 Department of Neuroinflammation, UCL Institute of Neurology, Queen Square Multiple Sclerosis Centre, University College London, London, UK

14 UMR 1064, INSERM, Centre de Recherche en Transplantation et Immunologie, Université de Nantes, Nantes, France

15 Service Neurologie, CHU Nantes, Nantes, France

16 Department of Neurology, CHU de Lille, University of Lille, Lille, France

17 Department of Neurology, University Hospital of Dijon, Dijon, France

18 Department of Neurology, CHU de Nancy, Nancy, France

19 Department of Neurology, CHU de Clermont-Ferrand, Clermont-Ferrand, France

20 Department of Neurology, Centre Hospitalier de Poissy, Saint Germain, France 
acute optic neuritis (AON) or slowly progressive optic neuropathy $(\mathrm{PON})$. The primary endpoint was the mean change from baseline to month 6 in VA measured in logarithm of the minimum angle of resolution $(\log M A R)$ at $100 \%$ contrast of the selected eye. Visually evoked potentials, visual field, retinal nerve fiber layer (RNFL) thickness, and health outcomes were also assessed.

Results Ninety-three patients received MD1003 $(n=65)$ or placebo $(n=28)$. The study did not meet its primary endpoint, as the mean change in the primary endpoint was nonsignificantly larger $(p=0.66)$ with MD1003 $(-0.061$ $\log$ MAR, +3.1 letters $)$ than with placebo $(-0.036$ logMAR, +1.8 letters). Pre-planned subgroup analyses showed that $100 \%$ contrast VA improved by a mean of + 2.8 letters $(-0.058 \log$ MAR) with MD1003 and worsened by -1.5 letters ( +0.029 logMAR) with placebo $(p=0.45)$ in the subgroup of patients with PON. MD1003-treated patients also had nonsignificant improvement in $\log \mathrm{MAR}$ at $5 \%$ contrast and in RNFL thickness and health outcome scores when compared with placebo-treated patients. There was no superiority of MD1003 vs placebo in patients with AON. The safety profile of MD1003 was similar to that of placebo.

Conclusions MD1003 did not significantly improve VA compared with placebo in patients with MS experiencing chronic visual loss. An interesting trend favoring MD1003 was observed in the subgroup of patients with PON. Treatment was overall well tolerated.

Trial registration EudraCT identifier 2013-002112-27. ClinicalTrials.gov Identifier: NCT02220244

Funding MedDay Pharmaceuticals.

21 INSERM 1434, Department of Neurology, Clinical Investigation Center, CHU de Strasbourg, Strasbourg, France

22 Department of Neurology, CHU de Caen, Caen, France

23 MedDay Pharmaceuticals, Paris, France

24 Department of Ophthalmology, Faculty of Medicine, CHU de Reims, URCA, Reims, France

\section{Key Points}

Assessment of optic nerve function is a readily accessible target for evaluating MS therapies that aim to restore neurological function. This study evaluated whether high-dose pharmaceutical-grade biotin (hdPB; MD1003) could improve visual function in patients with MS who had chronic optic neuritis $(\mathrm{ON})$

MD1003 did not improve visual function compared with placebo in the subset of patients with fixed visual loss following an acute episode of ON; however, there were trends towards improvement in a number of measures of visual function in patients with progressive chronic optic neuropathy

These results are consistent with recent data that showed that MD1003 can decrease progression and improving walking disability in patients with progressive MS. The treatment was overall well tolerated

\section{Introduction}

Multiple sclerosis (MS) is a frequent and disabling neurological disease characterized by multifocal myelin destruction in the central nervous system. Most patients with MS experience an initial period of relapsing-remitting disease (RRMS) followed later by a progressive disease course (secondary progressive MS; SPMS) [1]. Approximately $15 \%$ of patients experience progressive disease from onset (primary progressive MS; PPMS) [1, 2]. Progressive MS, either SPMS or PPMS, can be further categorized as active or non-active depending on the presence of superimposed inflammatory activity [3]. There are currently no disease-modifying therapies approved for the treatment of non-active progressive MS.

Among the potential causes of progressive axonal degeneration in progressive MS are chronic demyelination and mitochondrial dysfunction, which both lead to a state of virtual hypoxia [4-6]. We recently reported the results of a randomized, double-blind, placebo-controlled study that showed that MD1003 (high-dose pharmaceutical-grade biotin [hdPB]) achieved sustained reversal of MS-related disability in patients with progressive MS without activity in the last 2 years before inclusion [7]. Biotin is a cofactor for acetyl-CoA carboxylase, the rate-limiting enzyme that generates malonyl-CoA, the two-carbon building block for fatty acid synthesis, which is an essential component of myelin $[9,10]$. Biotin is also a cofactor for three enzymes 
that generate intermediates for the tricarboxylic acid cycle: pyruvate carboxylase, 3-methylcrotonyl-CoA carboxylase, and propionyl-CoA carboxylase [9]. Therefore, the improvements in disability seen in patients with progressive MS treated with hdPB may be due to an increase in the supply of precursors for fatty acid and myelin synthesis and/or replenishment of the pool of adenosine triphosphate (ATP) in hypoxic neurons [7, 8].

Acute optic neuritis (ON) is an inflammatory and demyelinating disorder of the optic nerve that occurs in up to $50 \%$ of patients with MS and is the first symptom in up to $20 \%$ [11, 12]. Optic neuritis leads to loss of vision and typically presents as an acute monocular loss of vision, though it can affect both eyes either simultaneously or sequentially $[13,14]$. Symptoms of ON include reduced visual acuity (VA), periocular pain (especially during eye movements), reduced contrast sensitivity, dysfunction of color vision, and visual field defects [11, 12]. Vision loss typically develops over a period of hours or days and peaks within 1 or 2 weeks [11]. Most patients regain VA within 6 months, though visual contrast often remains impaired $[15,16]$. In a minority of cases, VA remains low more than 6 months after an acute ON. This type of chronic visual loss is referred to as 'sequelae of an acute optic neuritis' (AON). In rare cases, visual loss becomes slowly progressive, socalled 'progressive optic neuropathy' (PON) [17-19].

Patients with optic nerve injury represent a good target group to evaluate the efficacy of drugs aimed at restoring neurological function in patients with MS, as the optic nerves are accessible to precise and quantitative measures. Visual function can readily be assessed using ETDRS (Early Treatment Diabetic Retinopathy Study) logarithm of the minimum angle of resolution $(\log \mathrm{MAR})$ charts at high and low contrast to determine VA [20] and automated perimetry to examine visual field defects [21]. Optic nerve function can be assessed by recording visual evoked potentials (VEPs) [22] and the pathological changes seen in the thickness of the retinal nerve fiber layer (RNFL) can be accurately quantified via optical coherence tomography (OCT).

In the initial open-label study of MD1003 in patients with progressive MS, four patients with a progressive visual loss caused by chronic ON (PON type) had an improvement in VA within 6 months of treatment with hdPB [23]. The aim of the MS-ON study was to confirm these initial observations in patients with either AON or PON.

\section{Methods}

\subsection{Study Design}

MS-ON was a 6-month double-blind, multi-center, randomized, placebo-controlled study followed by a 6-month extension phase during which all patients received MD1003 [Online Resources 1, see electronic supplementary material (ESM)]. The study was conducted between October 2013 and September 2015 at 19 sites in France and one site in the United Kingdom.

\subsection{Participants}

Eligible patients were aged 18-75 years old with a diagnosis of MS fulfilling the 2010 McDonald criteria [24], unior bilateral optic neuropathy with VA in the worst eye of $\leq$ $5 / 10$ (decimal scale; 20/40 in US customary units) confirmed at 6 months, and evidence of worsening VA during the last 3 years (defined as a change in VA of $\geq 1 / 10$ points or more than one line). In AON, VA had to remain stable at least in the 6 months prior to inclusion. In PON, progressive visual loss had to have been noted at two different visits in the previous 3 years.

Key exclusion criteria included relapse of ON within 3 months before inclusion; other concomitant ocular conditions (glaucoma, cataract, retinopathy, anterior uveitis, myopia $>7$ dioptrics, intraocular pressure $>20 \mathrm{mmHg}$, amblyopia, retinal or optic head abnormalities); bilateral $\mathrm{VA}<1 / 20$, visual impairment caused by ocular flutter or nystagmus; or normal RNFL. Patients who were treated with fampridine initiated $<1$ month prior to inclusion or any other new medication for MS (immunomodulators and immunosuppressive agents) initiated $<3$ months prior to inclusion were also excluded. Full eligibility criteria are shown in Online Resource 2 (see ESM).

\subsection{Intervention}

In the placebo-controlled phase of the trial, patients were randomized 2:1 to either MD1003 (oral biotin $300 \mathrm{mg} /$ day administered as 100-mg capsules three times daily) or placebo. Placebo capsules were identical to hdPB capsules except for an additional $100 \mathrm{mg}$ lactose excipient in place of hdPB. Randomization was performed with a computergenerated sequence provided by an independent contract research organization. Each participating hospital pharmacy was provided with a block of six treatment units (four active and two placebo treatments in random order). No stratification was performed.

Patients and investigators were masked to the assigned treatment during the placebo-controlled phase and remained blinded during the extension phase as to which treatment was administered during the first phase. The treating neurologist was different from the evaluating ophthalmologist.

All usual treatments were allowed during the study providing these were initiated as described in the exclusion criteria. 


\subsection{Assessments}

Impairment of the optic nerve was assessed by VA in each eye separately using ETDRS $\log$ MAR charts presented with standard illumination [20]. Visual acuity was performed at 100 and 5\% contrast at screening, baseline, and every 3 months.

In addition, VEPs, automated perimetry, and OCT including RNFL thickness was performed. Assessment of VEPs was conducted at baseline and months 6 and 12 according to guidelines of the International Society for Clinical Electrophysiology of Vision [25]. Improvement was defined as (i) the reappearance of a P100 wave not visible in a previous examination or (ii) improvement of the P100 wave latency of at least $10 \mathrm{~ms}$. Visual field analyses were performed using standard automated perimetry at screening, baseline, and months 6 and 12 . Spectral domain OCT was conducted at baseline and months 6 and 12 and RNFL thickness and macular volume were recorded at these time points.

Health outcome assessments consisted of the clinical global impression scale evaluated by the patient (SGI) and by the clinician (CGI) at months 6 and 12 and the Multiple Sclerosis Quality of Life-54 (MSQOL-54) questionnaire and the National Eye Institute 25-Item Visual Function Questionnaire (NEIVFQ-25) assessed at baseline, month 6, and month 12 .

A detailed description of all the efficacy and health outcome assessments used in this study is provided in the Online Resources (Online Resources 3, see ESM).

\subsection{Study Objectives}

The primary endpoint of the study was the mean absolute change from baseline to month 6 of VA in the selected eye as measured by best-corrected VA ( $\log$ MAR) at $100 \%$ contrast. The selected eye was the eye with the worst VA at baseline and with evidence of worsening during the past 3 years.

Secondary endpoints were assessed at month 6 and comprised the proportion of patients with improvement of VA of the selected eye of $\geq 0.3 \log$ MAR at $100 \%$ contrast or improvement of binocular VA from $<70$ to $\geq 70 / 100$ at $100 \%$ contrast; the proportion of eyes with reappearance of P100 waves or improvement of P100 latencies of $\geq 10 \mathrm{~ms}$; the mean change from baseline in selected P100 latencies and amplitudes (all eyes); CGI and SGI; and the mean change from baseline in NEIVFQ-25 composite score and MSQOL-54 composite score and sub-scores.

Exploratory analyses assessed at month 6 consisted of the mean change from baseline in logMAR at 100 and 5\% contrast in non-selected eyes, all eyes, and binocular vision (at $100 \%$ contrast only); the proportion of patients with improvement at $5 \%$ contrast in at least one eye of $\geq 0.3, \geq$ 0.2 , or $\geq 0.1 \log$ MAR; and the mean change from baseline of the mean deviation in visual fields, RNFL thickness, temporal RNFL values, and macula volume.

Safety was investigated by comparing the incidence of adverse events (AEs) and laboratory/electrocardiogram findings between study arms.

\subsection{Statistical Considerations}

Sample size determination was based on limited data. In four patients with progressive $\mathrm{ON}$ treated with hdPB $300 \mathrm{mg} /$ day [23], a mean improvement of VA on treatment of $0.423 \log$ MAR (SD 0.29) was observed. VA in patients with ON after 6 months of chronic visual loss does not typically improve spontaneously [26]. Therefore, assuming a conservative mean improvement of $0.3 \operatorname{logMAR}$ for the patients in the MD1003 arm and a mean change with placebo of $0.0 \log \mathrm{MAR}$, and a common standard deviation of $0.29,70$ patients in the MD1003 arm and 35 patients in the placebo arm (total sample size of 105) were required to provide $99 \%$ power to detect a difference between arms, at a 0.05 two-sided significance level.

Quantitative data were summarized using descriptive statistics and qualitative data using proportions. The intentto-treat population (ITT) was defined as all randomized patients who received at least one dose of study medication and had at least one baseline VA score. All patients from the ITT population who also had a VA score at month 6 without any major protocol deviations comprised the perprotocol population (PP). The safety population was defined as all patients who received at least one dose of study drug. Baseline values were defined as the last available assessments before or at date of first study drug administration.

The primary endpoint was assessed in the ITT population and differences in the mean absolute change in VA at month 6 between arms were evaluated by an ANCOVA analysis with adjustment for baseline VA. A Welch $t$ test and a Wilcoxon-Mann-Whitney test were performed on the primary efficacy endpoint in the ITT population as sensitivity analyses. The primary efficacy analysis was also assessed at months 3 and 12 in the ITT population and in the PP population. Pre-planned subgroup analyses were conducted in subgroups stratified according to PON or AON; baseline binocular VA at $100 \%$ contrast $<70$ or $\geq$ $70 / 100$; and baseline RNFL thickness $<75$ or $\geq 75 \mu \mathrm{M}$. Between the two groups, the differences in secondary endpoints were examined using Fisher's exact test for proportions, Mann-Whitney's $U$ test for means, and a logistic model using generalized estimating equations. All statistical analyses were two-sided with a significance level of $p<0.05$ and were conducted by the Biostatistics Unit of 
BIOTRIAL using SAS ${ }^{\circledR}$ software 9.3 (SAS Institute Inc., Cary, NC, USA). The Last Observation Carried Forward imputation method was used for missing data.

\section{Results}

Between October 2013 and January 2015, 117 patients were screened, of which 93 met the inclusion criteria and were randomized to MD1003 $(n=65 ; 69.9 \%)$ or placebo ( $n=28 ; 30.1 \%$; Fig. 1). Baseline patient demographics and disease characteristics were balanced between study arms (Table 1). Most patients $(62 ; 66.7 \%)$ had AON; 31 (33.3\%) patients had PON. Fifty-nine patients (63.4\%) had RRMS, 14 (15.1\%) had PPMS, and 20 (21.5\%) had SPMS. Patients were severely impaired as illustrated by low VA and neuro-ophthalmologic measures. Most (61/89; 68.5\%) patients did not have identifiable P100 waves as assessed by VEP and the majority $(74 / 81 ; 84.1 \%)$ had a thin $(<75 \mu \mathrm{m})$ RNFL. The distribution of disease-modifying concomitant medications was similar between arms during the placebo-controlled part of the study except for methylprednisolone and natalizumab (Table 1).
Treatment compliance was good during the placebocontrolled part with 67 (72.0\%) patients overall achieving compliance rates of $\geq 90 \%$. Mean duration of treatment in the safety population was 5.6 months in both arms. One patient was withdrawn from the study during the placebocontrolled part because of an adverse event (AE) - retinal artery occlusion in the MD1003 arm considered to be possibly treatment related by the Investigator. The remaining 92 patients entered the 6-month extension phase. Treatment compliance was lower during the extension phase with $55(59.8 \%)$ patients overall achieving compliance rates $\geq 90 \%$. The mean $(\mathrm{SD})$ overall duration of treatment during the extension phase was $5.5(0.6)$ months. Twelve $(23.7 \%)$ patients had treatment withdrawal during the extension phase (Fig. 1).

\subsection{Primary Efficacy Analysis}

At month 6, the mean (SEM) change in $\operatorname{logMAR} \mathrm{VA}$ at $100 \%$ contrast for the selected eye was $-0.061(0.026)$ in the MD1003 arm and $-0.036(0.035)$ in the placebo arm (Fig. 2), indicating an improvement in VA in both arms corresponding to an additional 3.1 letters and 1.8 letters,
Fig. 1 CONSORT diagramscreening, enrolment, randomization and follow-up of study patients

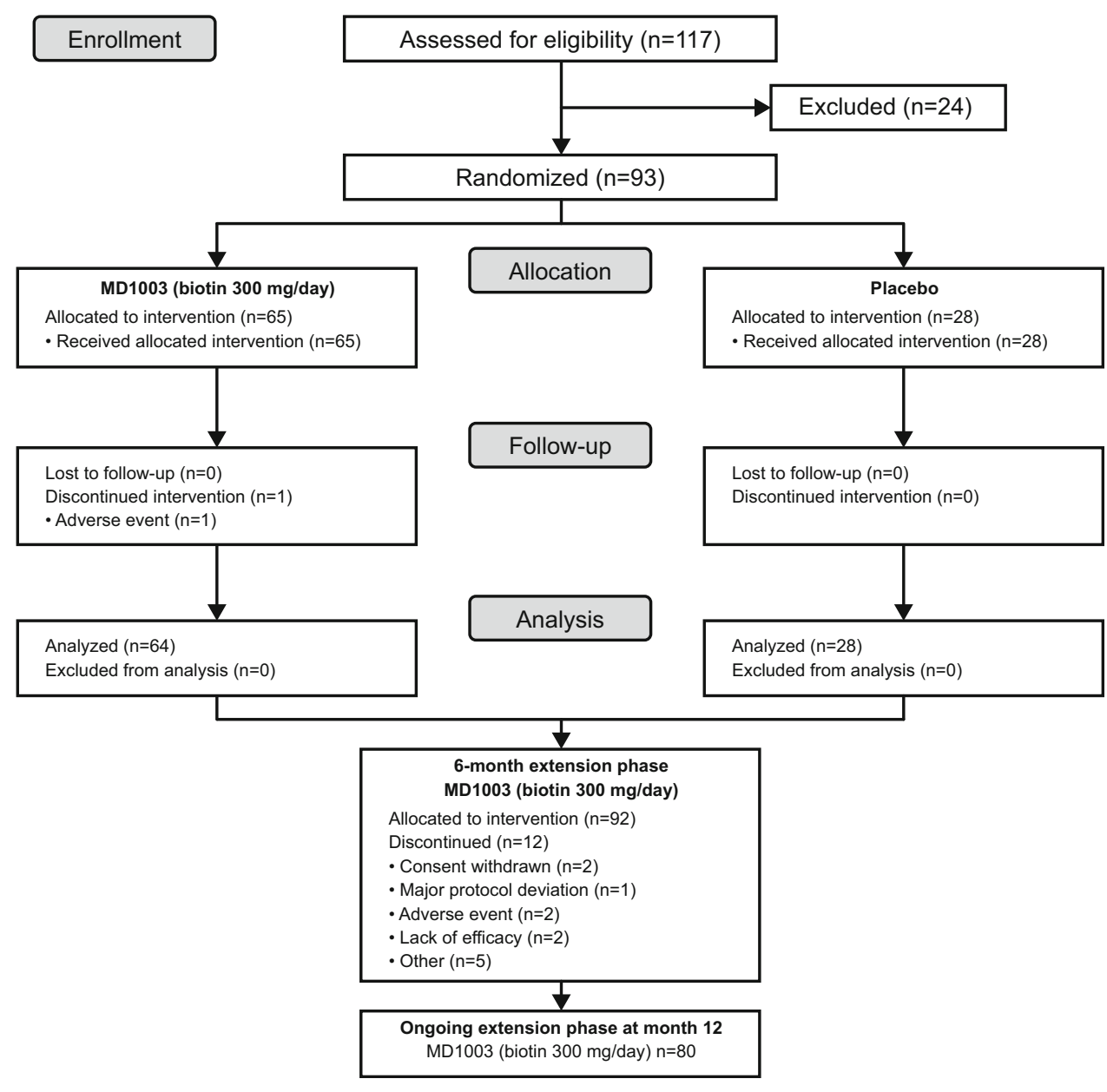


Table 1 Baseline demographics and disease characteristics (ITT population)

\begin{tabular}{|c|c|c|c|}
\hline & $\operatorname{MD} 1003(n=65)$ & Placebo $(n=28)$ & $p$ value \\
\hline Female, $n(\%)$ & $35(53.8)$ & $15(53.6)$ & 1 \\
\hline Age, years, mean (SD) & $41.6(10.5)$ & $41.1(10.6)$ & $0.795^{* *}$ \\
\hline Duration of MS (SD), years ${ }^{\mathrm{a}}$ & $12.6(9.4)$ & $11.3(8.1)$ & $0.705^{* *}$ \\
\hline Relapsing-remitting MS, $n(\%)^{\mathrm{f}}$ & $45(69.2)$ & $14(50.0)$ & 0.101 \\
\hline Progressive MS, $n(\%)^{\mathrm{f}}$ & $20(30.8)$ & $14(50.0)$ & 0.101 \\
\hline $\mathrm{AON}, n(\%)$ & $41(63.1)$ & $21(75.0)$ & 0.340 \\
\hline $\mathrm{PON}, n(\%)$ & $24(36.9)$ & $7(25.0)$ & 0.340 \\
\hline Mean VA in $\log$ Mar $(\mathrm{SD})^{\mathrm{b}}$ & $0.82(0.38)$ & $0.77(0.45)$ & $0.196^{* *}$ \\
\hline \multicolumn{4}{|l|}{ Binocular VA, $n(\%)^{\mathrm{b}, \mathrm{c}}$} \\
\hline$<70$ & $21(39.6)$ & $7(30.4)$ & 0.623 \\
\hline$\geq 70-100$ & $32(60.4)$ & $16(69.6)$ & 0.506 \\
\hline \multicolumn{4}{|l|}{ P100 waves, $n(\%)^{\mathrm{d}}$} \\
\hline Absent & $42(66.7)$ & $19(73.1)$ & 0.815 \\
\hline Present & $21(33.3)$ & $7(26.9)$ & 0.623 \\
\hline \multicolumn{4}{|l|}{ RNFL, $n(\%)^{\mathrm{e}}$} \\
\hline$<75 \mu \mathrm{M}$ & $51(83.6)$ & $23(85.2)$ & 0.784 \\
\hline$\geq 75 \mu \mathrm{M}$ & $10(16.4)$ & $4(14.8)$ & 1 \\
\hline Concomitant DMT (\%) & $46(70.7)$ & $16(57.1)$ & 0.234 \\
\hline Fampridine & $6(9.2)$ & $2(7.1)$ & 1 \\
\hline Fingolimod & $10(15.4)$ & $7(25.0)$ & 0.380 \\
\hline Interferon $\beta-1 \mathrm{~A}$ or $\beta-1 \mathrm{~B}$ & $10(15.4)$ & $3(10.7)$ & 0.747 \\
\hline Natalizumab & $9(13.8)$ & $1(3.6)$ & 0.272 \\
\hline Cyclophosphamide & $0(0.0)$ & $1(3.6)$ & 0.301 \\
\hline Methylprednisolone & $12(18.5)$ & $1(3.6)$ & 0.099 \\
\hline Glatiramer acetate & $9(13.8)$ & $4(14.3)$ & 1 \\
\hline Methotrexate & $1(1.5)$ & $2(7.1)$ & 0.214 \\
\hline Azathioprine & $2(3.1)$ & $1(3.6)$ & 1 \\
\hline Mycophenolate mofetil & $7(10.8)$ & $1(3.6)$ & 0.427 \\
\hline
\end{tabular}

$A O N$ acute optic neuritis, DMT disease-modifying therapy, ITT intention-to-treat, $O N$ optic neuritis, $M S$ multiple sclerosis, $P O N$ progressive optic neuropathy, $R N F L$ retinal nerve fiber layer, $S D$ standard deviation, $V A$ visual acuity

$p$ values were calculated using Fisher exact test or Mann-Whitney test $(* *)$

${ }^{\mathrm{a}}$ Time between first appearance of MS and first treatment

${ }^{\mathrm{b}}$ At $100 \%$ contrast; data were missing for: ${ }^{\mathrm{c}} 12$ patients in the MD1003 group and five patients in the placebo group, ${ }^{\mathrm{d}}$ two patients in each group, ${ }^{\mathrm{e}}$ four patients in the MD1003 group and one patient in the placebo group ${ }^{\mathrm{f}}$ The progressive and relapsing phenotypes relate to the overall patient status whereas the AON/PON classification relates to the optic neuropathy type. The two classifications do not necessarily correlate respectively, on the ETDRS chart. This difference between arms was not significant (mean treatment difference $-0.01983 ; 95 \%$ CI -0.1085 to $0.0689 ; p=0.66)$. At the end of the extension phase (month 12), the mean change in $\log$ MAR VA at $100 \%$ contrast for the selected eye corresponded to +4.3 letters in the MD1003 > MD1003 arm and +4.0 letters in the placebo $>$ MD1003 arm.

There was no difference between treatment arms in preplanned subgroup analyses according to fampridine treatment and in patients with $<70$ or $\geq 70 / 100$ binocular VA at $100 \%$ contrast at baseline, or in patients with $<75$ or $\geq 75 \mu \mathrm{M}$ RNFL thickness at baseline. All results were similar when analyzed in the PP population $(n=86)$.

\subsubsection{Subgroup Analyses in Acute Optic Neuritis (AON) and Progressive Optic Neuropathy (PON)}

In patients with $\mathrm{PON}$, the mean (SD) change from baseline in $\log$ MAR values at $100 \%$ contrast in the selected eye at month 6 was $-0.058(0.185)$ in the MD1003 group and + $0.029(0.176)$ in the placebo group. This equated to an improvement of +2.8 letters in the MD1003 group and a 


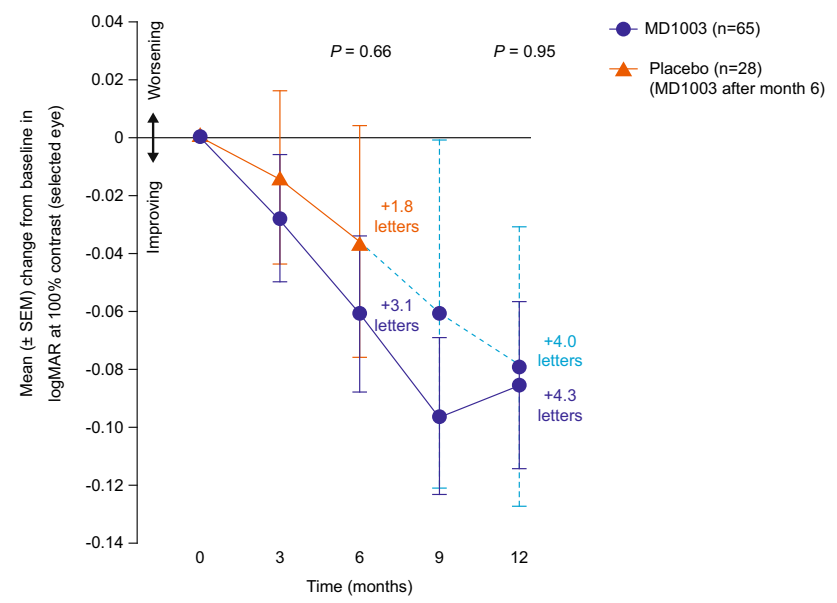

Fig. 2 Primary endpoint: mean ( \pm SEM) change from baseline in logMAR at $100 \%$ contrast (selected eye) at month $6(n=93)$. ITT population. The figure also shows the results of the primary efficacy analysis during the extension phase (month $12 ; n=92$ ). ITT intention-to-treat, SEM standard error of the mean worsening of -1.5 letters in the placebo group $(p=0.45)$ (Fig. 3a). At month 12, the mean change in logMAR values from baseline corresponded to +4.6 letters in the MD1003 group and -1.2 letters in the placebo group at month 12 $(p=0.26)$. In contrast, there was no difference between treatment groups in patients with AON (Fig 3b).

\subsection{Secondary and Exploratory Endpoints}

Overall, there were no significant differences between treatment arms for the secondary and exploratory endpoints (Online Resource 4, see ESM). Post hoc analyses according to $\mathrm{PON}$ and AON subgroups revealed trends in several secondary endpoints favoring MD1003 over placebo in patients with PON (Fig. 4), which was not the case in patients with AON. In the PON subgroup, the mean change from baseline to month 6 in $\log$ MAR at $5 \%$ contrast for all eyes improved in the MD1003 group (+ 3.7 letters) but
Fig. 3 Pre-planned subgroup analysis of the primary efficacy analysis in patients with a progressive optic neuropathy $(n=31)$ and $\mathbf{b}$ sequelae of an acute optic neuritis $(n=62)$. ITT population. ITT intentionto-treat, SEM standard error of the mean a Progressive optic neuropathy $(n=31)$

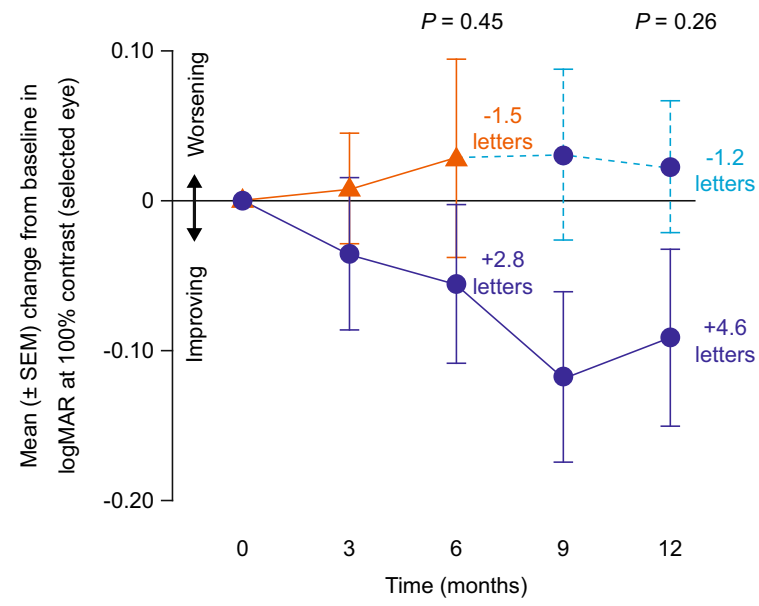

MD1003 ( $n=24)$

Placebo $(n=7)$ (MD1003 after month 6)

b Sequelae of an acute optic neuritis $(n=62)$

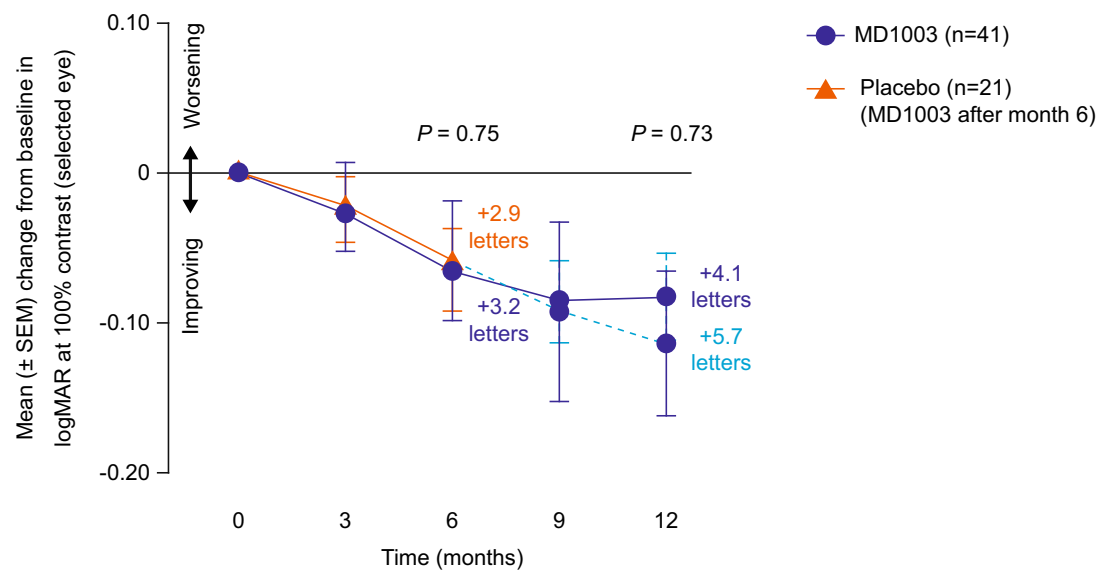



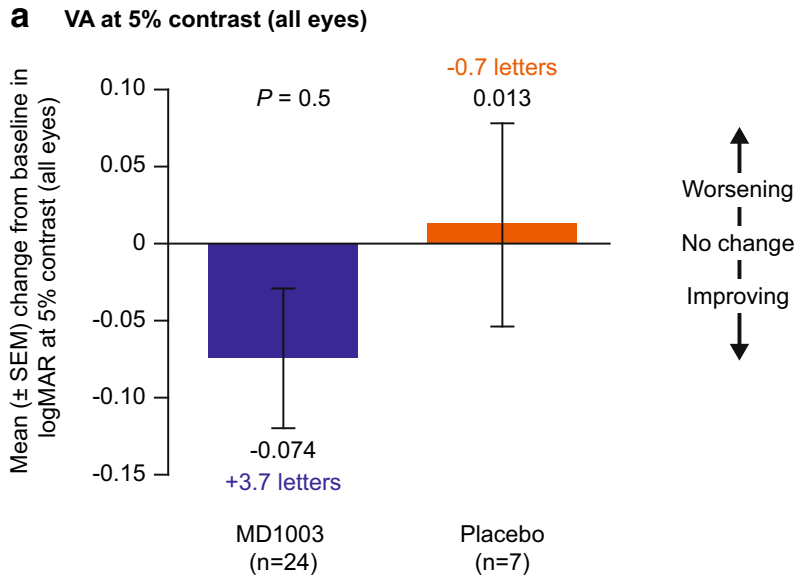

b RNFL thickness of all eyes
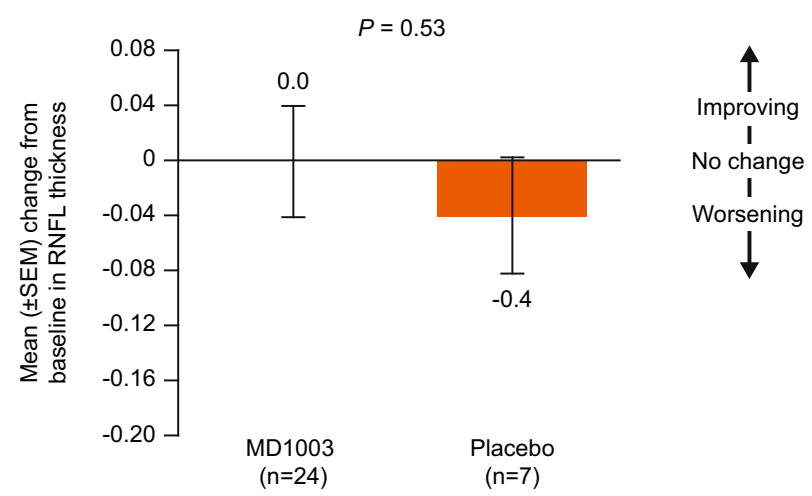

C NEIVFQ-25 composite score
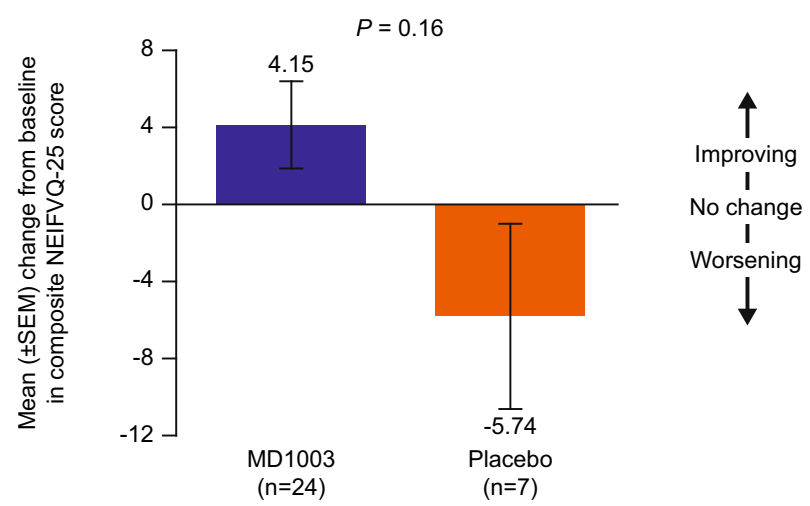

Fig. 4 Mean ( \pm SEM) change from baseline to month 6 in a $\log$ MAR at $5 \%$ contrast (all eyes), b mean RNFL thickness (all eyes), and c NEIVFQ-25 composite score, in patients with progressive optic neuropathy $(n=31)$. ITT population. ITT intention-totreat, NEIVFQ-25 National Eye Institute 25-Item Visual Function Questionnaire, $R N F L$ retinal nerve fiber layer, $S E M$ standard error of the mean

worsened in the placebo group ( -0.7 letters; Fig. 4a). MD1003 also appeared to halt the thinning of the RNFL in the PON subgroup during the 6-month placebo-controlled phase (Fig. 4b). The positive effect of MD1003 on vision in the subgroup of patients with PON was also reflected in the trend towards improvement in vision-associated daily life activities compared with placebo, as assessed by the NEIVFQ-25 questionnaire (Fig. 4c). It should be noted that, because of the low number of patients in the placebo group $(n=7)$, these positive trends are merely indicative of MD1003 effect.

There were no notable changes between study arms in the PON subgroup in visual field assessments or VEPs.

\subsection{Safety}

Treatment in both arms was well tolerated. During the placebo-controlled part of the study, a similar proportion of patients experienced an $\mathrm{AE}$ in both arms (49 [75.4\%] in the MD1003 arm and 22 [78.6\%] patients in the placebo arm; Table 2). Most AEs were mild to moderate in severity (95.4\% of patients in the MD1003 arm; 92.9\% of patients in the placebo arm). Severe AEs were recorded in three (4.6\%) patients in the MD1003 arm (single cases of apparent hyperthyroidism due to the known biotin-thyroid assay interaction, retinal artery occlusion, and back pain) and two $(7.1 \%)$ patients in the placebo arm (fatigue and gastroenteritis). The only serious AEs to occur in more than one patient were MS-related: MS relapse (symptoms confirmed to be associated with evolution of the disease) occurred in nine (13.8\%) MD1003-treated patients and one (3.6\%) placebo-treated patient, and MS symptoms (possibly but not necessarily associated with MS) occurred in two MD1003-treated patients. During the extension phase, 32 patients (50.0\%) in the MD1003 > MD1003 arm and 12 patients $(42.9 \%)$ in the placebo $>$ MD1003 arm experienced AEs. Again, the only serious adverse events (SAEs) reported in more than one patient were MS-related: MS relapse was recorded in three $(4.7 \%)$ patients in the MD1003 > MD1003 group and three (10.7\%) patients in the placebo $>$ MD1003 group. No deaths occurred during the study.

\section{Discussion}

We evaluated the efficacy of MD1003 in MS patients with chronic visual loss in a randomized, double-blind, placebocontrolled, 6-month study involving 93 patients, followed by a 6-month, open-label extension during which all patients received MD1003. Inclusion of both AON and PON allowed us to evaluate whether MD1003 would have efficacy in driving recovery after a relapse $(\mathrm{AON})$ or would more specifically demonstrate efficacy in the progressive disease state (PON).

Overall, the primary objective of study MS-ON was not reached: the improvement in the number of letters read on the ETDRS chart at month 6 was larger with MD1003 (+ 
Table 2 Adverse events reported during the study (safety population)

\begin{tabular}{|c|c|c|c|}
\hline Trial phase & Trial arm 1 & Trial arm 2 & $p$ value \\
\hline Double-blind, placebo-controlled phase & $\operatorname{MD} 1003(n=65)$ & Placebo $(n=28)$ & \\
\hline Any AE, $n(\%)$ & $49(75.4)$ & $22(78.6)$ & 0.797 \\
\hline \multicolumn{4}{|c|}{ AEs occurring in $\geq 5 \%$ of patients in either group } \\
\hline MS relapse & $9(13.8)$ & $1(3.6)$ & 0.272 \\
\hline Nasopharyngitis & $8(12.3)$ & $1(3.6)$ & 0.269 \\
\hline Urinary tract infection & $4(6.2)$ & $1(3.6)$ & 1 \\
\hline Headache & $3(4.6)$ & $3(10.7)$ & 0.360 \\
\hline Asthenia & $2(3.1)$ & $2(7.1)$ & 0.581 \\
\hline Gastroenteritis & $1(1.5)$ & $3(10.7)$ & 0.079 \\
\hline Dizziness & & $2(7.1)$ & \\
\hline Depression & & $2(7.1)$ & \\
\hline Anxiety & & $2(7.1)$ & \\
\hline Any severe $\mathrm{AE}^{\mathrm{a}}, n(\%)$ & $3(4.6)$ & $2(7.1)$ & 0.635 \\
\hline \multicolumn{4}{|c|}{ Severe AEs occurring in $\geq 1$ patient in either group } \\
\hline Any SAE, $n(\%)$ & $9(13.8)$ & $3(10.7)$ & 1 \\
\hline \multicolumn{4}{|c|}{ SAEs occurring in $\geq 1$ patient in either group } \\
\hline MS relapse & $9(13.8)$ & $1(3.6)$ & 0.272 \\
\hline Extension phase & $\operatorname{MD} 1003>\operatorname{MD} 1003(n=64)$ & Placebo $>$ MD1003 $(n=28)$ & \\
\hline Any $\mathrm{AE}, n(\%)$ & $32(50.0)$ & $12(42.9)$ & 0.651 \\
\hline \multicolumn{4}{|c|}{ AEs occurring in $\geq 5 \%$ of patients in either group } \\
\hline Headache & $1(1.6)$ & $3(10.7)$ & 0.082 \\
\hline MS relapse & $3(4.7)$ & $3(10.7)$ & 0.363 \\
\hline Edema peripheral & & $2(7.1)$ & \\
\hline Oropharyngeal pain & & $2(7.1)$ & \\
\hline Any severe $\mathrm{AE}^{\mathrm{b}}, n(\%)$ & $1(1.6)$ & $2(7.1)$ & 0.218 \\
\hline \multicolumn{4}{|c|}{ Severe AEs occurring in $\geq 1$ patient in either group } \\
\hline Any SAE, $n(\%)$ & $6(9.4)$ & $4(14.3)$ & 0.485 \\
\hline \multicolumn{4}{|l|}{ SAEs occurring in $\geq 1$ patient in either group } \\
\hline MS relapse & $3(4.7)$ & $3(10.7)$ & 0.363 \\
\hline
\end{tabular}

$p$ values were calculated using Fisher exact test

$A E$ adverse event, $M S$ multiple sclerosis, $S A E$ serious adverse event

${ }^{a}$ Severe AEs during the placebo-controlled phase were single cases of hyperthyroidism, retinal artery occlusion, back pain, and MS relapse in the MD1003 group and single cases of fatigue and gastroenteritis in the placebo group

${ }^{\mathrm{b}}$ Severe AEs during the extension phase were a single case of joint dislocation in the MD1003 > MD1003 group and single cases of headache and MS relapse in the MD1003 > placebo group

3.1 letters) than with placebo $(+1.8$ letters $)$, but this difference was not statistically significant $(p=0.66)$. There were no significant overall differences between study arms in the secondary endpoints. These results might reflect in part the main limitations of our study. As mentioned earlier, sample size determination was based on limited data and the number of patients randomized was low, especially in the placebo group $(n=28)$. This small sample size limitation was further accentuated by the heterogeneity of the population, which included subsets of patients with two distinct types of ON (AON and PON). The fact that, as an average, placebo-treated patients with AON spontaneously improved during the course of the trial, led to potential underestimation of MD1003 therapeutic effect. In addition, the trial lasted only 6 months, which might have been too short to observe the full extent of MD1003 therapeutic potential.

Interestingly, the pre-planned subgroup analyses in patients with AON or PON revealed two important findings. There was clearly no effect of MD1003 in the subgroups of patients with $\mathrm{AON}$ for all measures related to 
optic nerve involvement. This suggests that MD1003 does not trigger or re-initiate recovery after a relapse in this population and time frame. In contrast, a consistent trend suggesting treatment efficacy was observed in the PON subgroup. This trend favoring MD1003 in patients with PON was observed for VA at $100 \%$ contrast, VA at $5 \%$ contrast, RNFL, and NEIVFQ-25. We observed no changes in VEPs or visual field in the subgroup of patients with PON. It should be noted that patients recruited to the study had considerable neurodegenerative damage of the optic nerve at baseline as evidenced by the absence of measurable P100 waves in approximately $70 \%$ of patients. Patients also had severe impairment of their visual field at baseline. This degree of baseline disability may have precluded any meaningful improvement in these measures of optic nerve function.

The high proportion of patients with AON recruited to the study (63.4\% of the overall population) may explain the failure of the study to reach the primary endpoint. In fact, only a relatively small number of patients with PON $(n=31)$ were recruited, reflecting the relative rarity of patients with this condition.

Since MD1003 is believed to target the underlying progressive aspect of MS [8], patients with PON provide an appropriate model for the assessment of efficacy of MD1003. The trend of efficacy observed in patients with PON is consistent with observations from a pilot open-label study in which VA improved after treatment with hdPB in four patients with PON [23]. This trend is also in line with the findings of study MS-SPI, which demonstrated that MD1003 significantly improved MS-related disability in patients with a progressive spinal form of MS [7]. Together, these data are consistent with the proposed mechanism of action for MD1003 in targeting mechanisms to improve neuronal metabolism in context of virtual hypoxia, consistent with the pathophysiology of progressive MS.

The absence of superiority vs placebo in patients with AON suggests that MD1003 does not accelerate spontaneous recovery after an acute optic neuritis in a relatively short (6 months) time frame. This finding suggests that MD1003 has some selective efficacy when progression is ongoing. Further to this, it should be noted that the frequency of patients with progressive MS was higher in the placebo (50\%) than in the MD1003 group (31\%), which might have negatively influenced the results of our study. The MD1003 group had a higher proportion of patients on natalizumab or methylprednisolone than had the placebo group. Since it is thought to promote remyelination [27], we cannot exclude a potential influence of natalizumab on the results of our study. However, that influence would have been likely very limited, given the low proportion of patients treated with natalizumab $(13.8 \%$ in the MD1003 group and $3.6 \%$ in the placebo group) and the fact that the treatment with natalizumab had to be introduced at least 6 months prior to the MS-ON trial. Fingolimod, which is also thought to induce remyelination [28], could have had an opposite influence to that of natalizumab, since the MD1003 group had a lower proportion of patients on fingolimod (15.4\%) than had the placebo group (25\%).

This study also confirmed the good safety and tolerability profile of MD1003. The safety profile of MD1003 was similar to that of placebo and consistent with data from the MS-SPI study [7] and the open-label pilot study [23]. The incidence of MS relapse was higher in the MD1003 group ( 9 of 65 patients; $13.8 \%$ ) than in the placebo group ( 1 of 28 patients; $3.6 \%$ ) during the double-blind phase of the study, and was also higher in the patients newly treated with MD1003 in the extension phase ( 3 of 28 patients; $10.7 \%$ ) than in the placebo group in the double-blind phase (1 of 28 patients; $3.6 \%$ ). Others have reported an increase in MS relapse in patients treated with high-dose biotin $[29,30]$; however, these studies used a different source and dosage of biotin than our study, which does not allow any generalization [31]. Branger et al. [32] reported an occurrence of relapse in five patients with progressive MS after 3-7 months of treatment with hdPB, and recommended close monitoring of lesions by MRI. In a post hoc analysis from the MS-SPI study in patients with progressive MS, the annualized MS relapse rate up to 36 months did not appear to be influenced by exposure to MD1003 [33]. We believe that in the present study, the number of patients with relapse is too low to allow us to draw reliable conclusions. It should be further noted that the incidence of MS relapse decreased in patients who continued to receive MD1003 during the 6-month extension phase (4.7\%) (Table 2). Additional safety data from an ongoing clinical trial on MD1003 (SPI2 study) may help clarify this issue.

\section{Conclusion}

While the efficacy endpoints of study MS-ON were not met, signs of efficacy were apparent in the subgroup of patients with progressive disease. These data provide further evidence that the most appropriate target group for MD1003 is patients with progressive forms of MS, a population that represents a significant unmet medical need. Our study could also provide useful insights on the limitations associated with the assessment of any therapeutic agent in patients with MS-associated chronic optic neuropathy.

\subsection{Previous presentation of data}

This work has been presented in part at the 68th American Academy of Neurology Annual Meeting, Vancouver, 
Canada (15-21 April 2016). Tourbah et al. Neurology 2016;86(16 Suppl.):S49.005

Acknowledgements This study, and editorial support for the preparation of this manuscript, was funded by MedDay Pharmaceuticals. We thank all patients and their families for their participation in this study and the entire study team. We also thank Jamie Ashman, PhD, of Prism Ideas for medical writing support. We also acknowledge the contribution of Floriana DeAngelis, University College London, Marie Braisher, University College London, Charlotte Rawlinson, University College London Hospitals NHS Foundation Trust, and Lucas B. Kipp, Stanford University, as study investigators.

\section{Compliance with ethical standards}

Funding This study, and editorial support for the preparation of this manuscript as well as open access fee, was funded by MedDay Pharmaceuticals.

Conflict of interest Ayman Tourbah received consulting and review activities fees and support for travel to meetings from MedDay Pharmaceuticals. Ahmed Toosy received speaker honoraria from Biomedia, Serono Symposia International Foundation, and Bayer, and meeting expenses from Biogen Idec. He is the local principal investigator for clinical trials in multiple sclerosis funded by MedDay Pharmaceuticals. Oliver Gout received personal compensation for scientific advisory boards and funding for travel and/or speaker honoraria from Biogen Idec, Novartis, Sanofi-Genzyme, Merck-Serono, MedDay, and Teva. David-Axel Laplaud received grants, consultancy fees or honoraria, and payment for lectures including service on speaker's bureaus from Merck, Biogen, Sanofi-Genzyme, Roche, and Novartis. Alain Vighetto, Christine Lebrun-Frenay, Jérôme De Sèze, and Carl Arndt received consultancy fees or honoraria from MedDay Pharmaceuticals. Jean Pelletier received unconditional research grants from Merck, Novartis, and Roche; consulting fees or honorarium from Biogen, Genzyme, MedDay Pharmaceuticals, Merck, Novartis, Roche, and Teva; and support for travel to meetings for the study, manuscript preparation or other purposes from Biogen, Genzyme, MedDay Pharmaceuticals, Merck, Novartis, Roche, and Teva. Pierre Clavelou received consulting fees or honoraria from Biogen, Roche Merck, Teva, Novartis, and Genzyme. Frédéric Sedel is Chief Executive Officer and Co-Founder of MedDay Pharmaceuticals. Giles Defer received personal compensation for scientific advisory boards and funding for travel and/or speaker honoraria from Biogen Idec, Novartis, Sanofi-Genzyme, Merck-Serono, and Teva; and institutional research grants from Merck-Serono, Biogen Idec, Sanofi-Genzyme, and Novartis. Véronique Deburghgraeve, Caroline Papeix, Pierre Labauge, David Brassat, Olivier Outteryck, Thibault Moreau, Marc Debouverie, and Olivier Heinzlef have nothing to declare.

Ethical standards All procedures performed in studies involving human participants were in accordance with the ethical standards of the institutional and/or national research committee and with the 1964 Helsinki declaration and its later amendments. The conduct of the study was overseen by an independent data safety monitoring board.

Informed consent All subjects gave their written informed consent prior to their inclusion in the study.

Open Access This article is distributed under the terms of the Creative Commons Attribution-NonCommercial 4.0 International License (http://creativecommons.org/licenses/by-nc/4.0/), which permits any noncommercial use, distribution, and reproduction in any medium, provided you give appropriate credit to the original author(s) and the source, provide a link to the Creative Commons license, and indicate if changes were made.

\section{References}

1. Confavreux C, Vukusic S, Moreau T, et al. Relapses and progression of disability in multiple sclerosis. $\mathrm{N}$ Engl $\mathrm{J}$ Med. 2000;343:1430-8.

2. Koch M, Kingwell E, Rieckmann P, et al. The natural history of primary progressive multiple sclerosis. Neurology. 2009;73:1996-2002.

3. Lublin FD. New multiple sclerosis phenotypic classification. Eur Neurol. 2014;72:1-5.

4. Levin MC, Douglas JN, Meyers L, et al. Neurodegeneration in multiple sclerosis involves multiple pathogenic mechanisms. Degener Neurol Neuromuscul Dis. 2014;4:49-63.

5. Stys PK, Zamponi GW, van MJ, et al. Will the real multiple sclerosis please stand up? Nat Rev Neurosci. 2012;13:507-14.

6. Trapp BD, Stys PK. Virtual hypoxia and chronic necrosis of demyelinated axons in multiple sclerosis. Lancet Neurol. 2009;8:280-91.

7. Tourbah A, Lebrun-Frenay C, Edan G, et al. MD1003 (high-dose biotin) for the treatment of progressive multiple sclerosis: a randomised, double-blind, placebo-controlled study. Mult Scler J. 2016;22:1719-31.

8. Sedel F, Bernard D, Mock DM, et al. Targeting demyelination and virtual hypoxia with high-dose biotin as a treatment for progressive multiple sclerosis. Neuropharmacology. 2015;110:644-53.

9. Tong L. Structure and function of biotin-dependent carboxylases. Cell Mol Life Sci. 2013;70:863-91.

10. DeWille JE, Horrocks LA. Synthesis and turnover of myelin phospholipids and cholesterol. In: Martensson RE, editor. Myelin: biology and chemistry. Boca Raton: CRC Press; 1992.

11. Balcer LJ. Clinical practice. Optic neuritis. N Engl J Med. 2006;354:1273-80.

12. Hoorbakht H, Bagherkashi F. Optic neuritis, its differential diagnosis and management. Open Ophthalmol J. 2012;6:65-72.

13. Hickman SJ, Dalton CM, Miller DH, et al. Management of acute optic neuritis. Lancet. 2002;360:1953-62.

14. Pirko I, Blauwet LA, Lesnick TG, et al. The natural history of recurrent optic neuritis. Arch Neurol. 2004;61:1401-5.

15. Wilhelm $\mathrm{H}$, Schabet M. The diagnosis and treatment of optic neuritis. Dtsch Arztebl Int. 2015;112:616-25.

16. Beck RW, Gal RL, Bhatti MT, et al. Visual function more than 10 years after optic neuritis: experience of the optic neuritis treatment trial. Am J Ophthalmol. 2004;137:77-83.

17. Frohman EM, Frohman TC, Zee DS, et al. The neuro-ophthalmology of multiple sclerosis. Lancet Neurol. 2005;4:111-21.

18. Ormerod IE, McDonald WI. Multiple sclerosis presenting with progressive visual failure. J Neurol Neurosurg Psychiatry. 1984;47:943-6.

19. Miller NR, Newman NJ, Biousse V, Kerrison JB. Walsh \& Hoyt's clinical neuro-ophthalmology: the essentials. 2nd ed. Philadelphia: Lippincott Williams Wilkins; 2007.

20. Shamir RR, Friedman Y, Joskowicz L, et al. Comparison of Snellen and early treatment diabetic retinopathy study charts using a computer simulation. Int J Ophthalmol. 2016;9:119-23.

21. Frisen L. Identification of functional visual field loss by automated static perimetry. Acta Ophthalmol. 2014;92:805-9.

22. Mienberg O, Flammer J, Ludin HP. Subclinical visual field defects in multiple sclerosis. Demonstration and quantification with automated perimetry, and comparison with visually evoked potentials. J Neurol. 1982;227:125-33. 
23. Sedel F, Papeix C, Bellanger A, et al. High doses of biotin in chronic progressive multiple sclerosis: a pilot study. Mult Scler Relat Disord. 2015;4:159-69.

24. Polman CH, Reingold SC, Banwell B, et al. Diagnostic criteria for multiple sclerosis: 2010 revisions to the McDonald criteria. Ann Neurol. 2011;69:292-302.

25. Odom JV, Bach M, Brigell M, et al. ISCEV standard for clinical visual evoked potentials (2009 update). Doc Ophthalmol. 2010;120:111-9.

26. Beck RW, Cleary PA, Anderson MM Jr, et al. A randomized, controlled trial of corticosteroids in the treatment of acute optic neuritis. The Optic Neuritis Study Group. N Engl J Med. 1992;326:581-8.

27. Zivadinov R, Dwyer MG, Hussein S, Carl E, Kennedy C, Andrews M, Hojnacki D, Heininen-Brown M, Willis L, Cherneva $\mathrm{M}$, Bergsland N, Weinstock-Guttman B, et al. Voxel-wise magnetization transfer imaging study of effects of natalizumab and IFN $\beta-1 \mathrm{a}$ in multiple sclerosis. Mult Scler. 2012;18:1125-34.

28. Miron VE, Ludwin SK, Darlington PJ, Jarjour AA, Soliven B, Kennedy TE, Antel JP. Fingolimod (FTY720) enhances remyelination following demyelination of organotypic cerebellar slices. Am J Pathol. 2010;176:2682-94.

29. Birnbaum G, Stulc J. High dose biotin as treatment for progressive multiple sclerosis. Mult Scler Relat Disord. 2017;18:141-3.

30. Granella F, Tsantes E, Siena E, Curti E. Breakthrough disease under high-dose biotin treatment in progressive multiple sclerosis. Mult Scler. 2017;23(S3):85-426 (P750).

31. Tourbah A, Sedel F. Letter to the Editors-Multiple sclerosis and related disorders-Birnbaum et al., 2017. Mult Scler Relat Disord. 2018;20:204.

32. Branger P, Derache N, Kassis N, Maillart E, Assouad R, Defer G. Relapses during high doses of biotin in progressive multiple sclerosis: a case series. Neurology 2018 (70th AAN annual meeting, Los Angeles, CA).

33. Lasser R, Sedel F, Brion G, Patterson J, Tourbah A. Annual relapse rates (ARR) in patients with spinal progressive multiple sclerosis treated with MD1003 (high-dose pharmaceutical biotin). Mult Scler. 2018;24(S1):11-117 (P045). 